\title{
RP part surface quality versus build orientation: when the layers are getting thinner
}

\author{
Yonghua Chen • Jianan Lu
}

Received: 29 December 2011 / Accepted: 3 September 2012 /Published online: 21 September 2012

(C) The Author(s) 2012. This article is published with open access at Springerlink.com

\begin{abstract}
Surface quality is a very important factor to be considered in determining part build orientation in rapid prototyping (RP) processes. Previous research has shown that parts built with inclined planes or curved surfaces along the build orientation have large staircase effect, thus have higher surface roughness compared to parts built with only vertical surfaces. However, as layers are getting thinner in rapid prototyping processes, the opposite might be true. In this study, a number of experiments and measurements are conducted first. In a single machine setup, two cylinders are built, one along axial direction and the other along transverse direction using an Objet ${ }^{\circledR}$ machine. Measurements have shown that surface roughness of RP parts built along the transverse direction is better than those from the axial direction. Through analysis and observation, the authors can conclude that when layers are small enough, surface curvature or slope along the build orientation may no longer be a major concern for RP part surface quality. Instead, the authors have observed that on-the-layer contour layout may cause even more serious surface quality problem. In other words, surface quality is not only dependent on build orientation, but more on scanning orientation on layers.
\end{abstract}

Keywords Rapid prototyping - Build orientation · Surface quality $\cdot$ Scanning orientation

Y. Chen $(\bowtie) \cdot$ J. Lu

Department of Mechanical Engineering,

The University of Hong Kong,

Pokfulam Road,

Hong Kong, China

e-mail: yhchen@hku.hk

\section{Introduction}

Rapid prototyping (RP) usually refers to technologies that build parts layer by layer rapidly without geometry constraints. Since its first commercial introduction in the late 1980s, there has been a widespread use of RP technologies in design visualization and prototyping, direct digital manufacturing, medical engineering, etc. [1]. However, RP parts have many problems that limit their applications. One of the problems is the surface quality of RP parts that is normally not up to the requirements of many applications.

Given the limitation of current RP technologies, researchers have focused on optimizing process parameters such as the build orientation to ensure good RP part surface quality [2]. In fact, build orientation is critical not only for part surface quality, but also for production time, the need of support structure, and the production cost. In this research, the build orientation with respect to only surface quality will be studied as it is perhaps the most single important factor in determining build orientation [3].

Different RP technology may have different factors that affect surface quality. For example, stereolithography apparatus (SLA) has a notable phenomenon called print-through on down-facing planes. This effect may cause the need for complimentary surface smoothing processes [4]. In general, the determination of build orientation needs to consider many factors such as part cost, build time, surface quality, overhang area, and support volume or area $[5,6]$. Xu et al. have reported the optimal build orientation selection for four different RP technologies [7], namely, SLA, selective laser sintering (SLS), fused deposition modeling, and laminated object manufacturing. Campbell et al. have presented a 
Fig. 1 The cylindrical model and its two build orientations; a the cylindrical model, $\mathbf{b}$ axial orientation, $\mathbf{c}$ transverse orientation

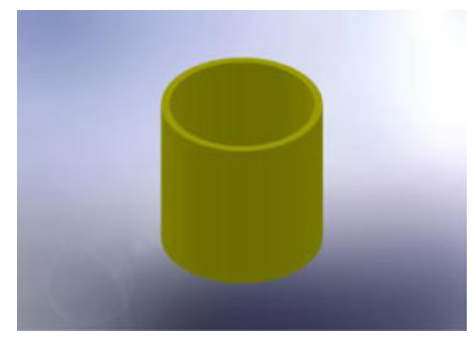

(a) the cylindrical model

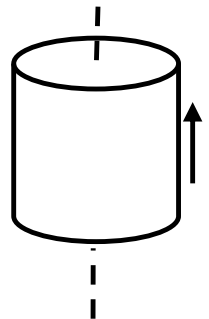

(b) axial orientation

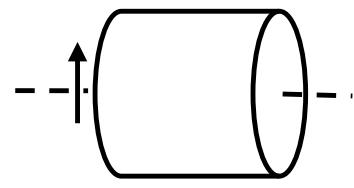

(c) transverse orientation
Fig. 2 Expected rough surface areas and the actual built models. a Expected rough surface areas. b Two built parts

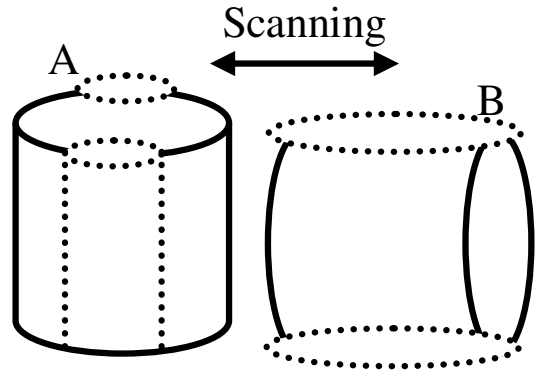

(a) Expected rough surface areas

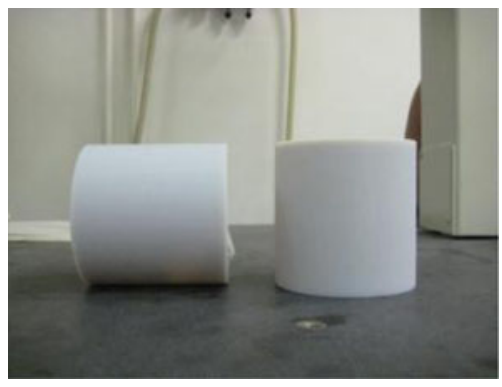

(b) Two built parts

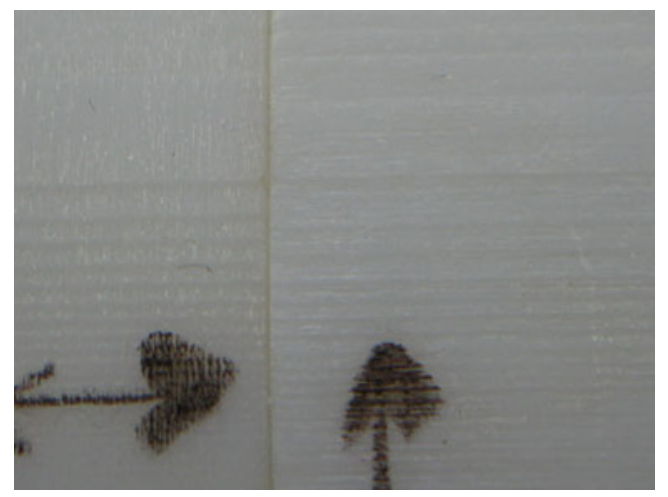

Fig. 3 A close view of the rough surface areas surface roughness visualization system for RP parts that are built in different orientation so that the best orientation with respect to the aimed applications could be identified [8]. A good review of build orientation can be found in [9].

In fact, effort in optimizing the build orientation for optimal surface quality has never stopped as can be seen in [10-13]. Almost all previous research on optimal build orientation refers the orientation of the object as a whole. In this research, it is argued that as the layer thickness is getting thinner with the everdeveloping RP technologies, the orientation of the object is no longer the major factor affecting part surface quality, instead it is found that the scanning orientation on layers that influence more on part surface quality. In order to clarify this finding, a number of experiments and measurements are presented first.

\section{Experiments}

Over the past a few years, the authors have built a lot of prototype parts or subsystems using an Objet Eden350. From observation, it was found that surface 
Fig. 4 Roughness

measurement set-up. a Axial measurements. b Transverse measurements

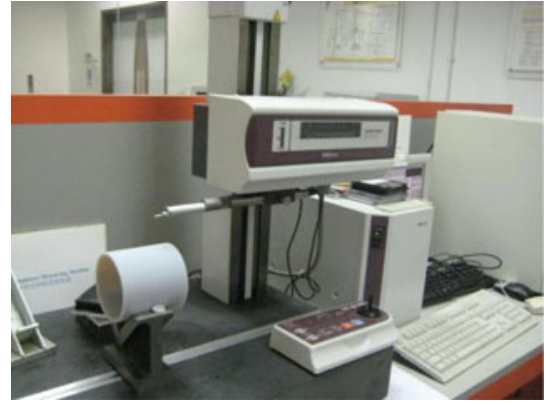

(a) Axial measurements

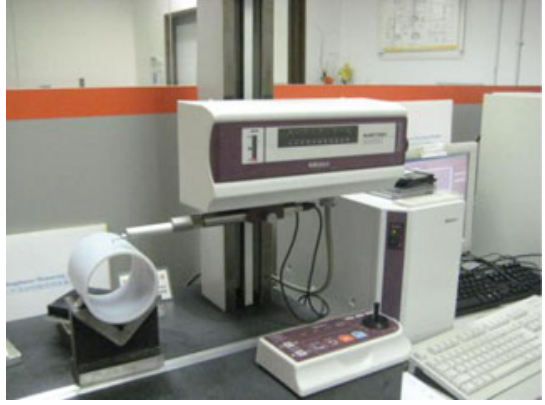

(b) transverse measurements roughness is no longer dependent mainly on build orientation as have been previously reported. Instead, the contour layout on layers may have a more dominant effect on the surface quality. It is argued that surface quality is dependent not only on the build orientation, but also on the scanning orientation on layers. In this paper, an experiment is first presented to show the effects of build orientation and scanning orientation to surface roughness. For easy illustration, two hollow cylinders are built in a single machine set up. The cylinders are defined by outer diameter $(100 \mathrm{~mm})$, height $(100 \mathrm{~mm})$, and wall thickness $(5 \mathrm{~mm})$. The outer diameter and height are set equal because the build process for the two cylinders (one built in axial orientation and the other in transverse orientation) can be completed at the same time. Furthermore, the part surface curvature change when built along the transverse direction is the same as the on the layer contour curvature change when built in axial orientation. Figure 1 shows the cylinder model and its two build orientations. For good surface quality, all previous studies will recommend the build orientation in Fig. 1b. That is, the axial orientation. In this study, the authors have experimentally found that the surface quality built in the transverse orientation as in Fig. 1c is better in terms of surface quality. This is mainly because the RP machine Objet Eden350 [14] used in the experiments has a very fine layer thickness of $16 \mu \mathrm{m}$, perhaps the finest among all available RP machines. Compared to the fine layer thickness, $x$ - and $y$-resolutions of the machine are relatively bigger, both are $42 \mu \mathrm{m}$. In fact, SLA technology based machines are also building part with thinner layers in recent years, say around $30 \mu \mathrm{m}$ [15]. That is, as the layers are getting thinner, the surface quality may no longer be primary determined by build orientation, but also by scanning orientation on layers. Material used in this experiment is VeroGray ${ }^{\mathrm{TM}}$.

When building the two cylinders, one was built along the axial direction and the other in transverse direction. Before fabrication, the authors can anticipate that the surface with the highest roughness values are as in the cylindrical areas enclosed in dashed lines in Fig. 2a. Label A shows the expected rough area from axial orientation while $\mathrm{B}$ indicates the rough area from transverse orientation. After fabrication as shown in Fig. 2b, it could be proved that the expectation is correct even by visual inspection using naked eyes. Figure 3 shows an enlarged image of A area (on the left) and B area (on the right). It can be seen from Fig. 3 that the staircase effects are both visible for part built from axial and transverse orientations. Even though, the A area looked a bit rougher, yet it is better to use quantitative measurements as the basis for final judgment.

The surface roughness was measured using a Surtronic 25 surface roughness tester from Taylor Hobson, as per DIN EN ISO 4288/ASME B461 and manufacturer's recommendation.
Fig. 5 Definition of measurement points. a Axial measurements. b Transverse build orientation. c 8 measurement points

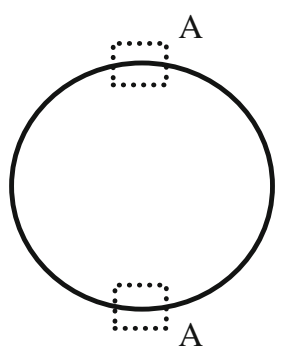

(a) Axial build orientation

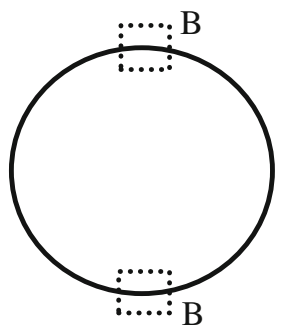

(b) Transverse build orientation

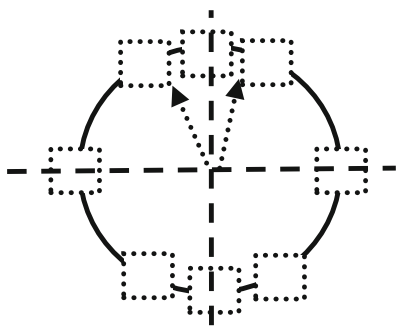

(c) 8 measurement points 
Table 1 Measurement parameter setting

\begin{tabular}{ll}
\hline Measurement length & $3.00 \mathrm{~mm}$ \\
Sample length & $0.25 \mathrm{~mm}$ \\
No. of sample & 12 \\
Pitch & $0.2 \mu \mathrm{m}$ \\
Lc & $0.25 \mathrm{~mm}$ \\
Filter & Gaussian \\
Pre-travel & $0.4 \mathrm{~mm}$ \\
Post-travel & $0.125 \mathrm{~mm}$ \\
Speed & $0.2 \mathrm{~mm} / \mathrm{s}$ \\
No. of points & 17625 \\
\hline
\end{tabular}

The most frequently used surface roughness parameter $\left(R_{a}\right)$ is used for comparison in all measurements in this paper. Figure 4 shows the measurement setup. For each predefined measurement position, two measurements will be done: one along the axial direction (Fig. 4a) and the other along the transverse direction (Fig. 4b). For each cylinder, eight points are defined as in Fig. 5c. They are mostly defined around the expected rough areas marked as A and B in Fig. 5a and b. The $\mathrm{A}$ and $\mathrm{B}$ areas are defined the same as that in Fig. 2a. Table 1 shows the measurement parameter settings.

2.1 Measurements for cylinder from transverse build orientation

Figure 6a shows measurement directions for the cylinder from transverse build orientation. The measurement points are labeled in Fig. 6b. Measurement results are tabulated in Table 2. Table $2 \mathrm{a}$ is the results for measurement along the axial direction (or the part grain direction), it can be seen that the roughness values are very small and smooth. Table $2 \mathrm{~b}$ records roughness values measured in the transverse direction. The roughness measurement values have large variations, with a maximum $R_{a}$ of $8.502 \mu \mathrm{m}$. Figure 7 shows the measurement chart with the largest $R_{a}$.

2.2 Measurements for cylinder from axial build orientation

Figure 8a shows measurement directions for the cylinder from axial build orientation. The measurement points are labeled in Fig. 8b. Measurement results are tabulated in Table 3. Table $3 \mathrm{a}$ is the results for measurement along the axial direction, it can be seen that the roughness values are very big, and the largest value is $14.787 \mu \mathrm{m}$. Table $3 \mathrm{~b}$ records roughness values measured in the transverse direction. The roughness measurement values have relatively small variations, with a maximum $R_{a}$ of $8.675 \mu \mathrm{m}$.

Table 4 is a summary of all measurements. It can be seen that the cylinder built from axial orientation has a larger surface roughness. And the surface roughness value of $8.675 \mu \mathrm{m}$ due to on the layer scan orientation is bigger than that of due to part orientation which is $8.502 \mu \mathrm{m}$. Of course, the difference is very small. This result can at least tell that on-the-layer-scan orientation has an equally big impact to surface quality as the part build orientation.

\subsection{Experiments with elliptical cylinder}

For the above experiments with cylinders in axial build orientation, all layer contours are the same and symmetrical. The scanning direction on layers makes no difference in
Fig. 6 Measurements for transverse build direction

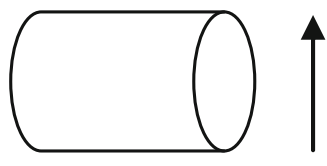

Build Orientation

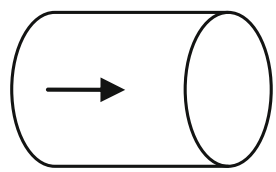

A: axial

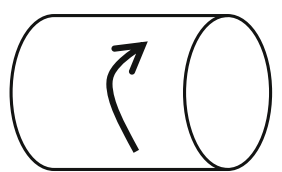

B: transverse (a) Measurement directions

(b) Measurement points 
Table 2 Measurement results for transverse build orientation

\begin{tabular}{ll}
\hline Points $\quad$ Roughness $R_{a}(\mu \mathrm{m})$ \\
\hline
\end{tabular}

\begin{tabular}{ll} 
(a) Axial direction measurement & \\
P1 & 1.392 \\
P2 & 0.728 \\
P10 & 2.312 \\
P9 & 3.122 \\
P15 & 2.973 \\
P14 & 2.160 \\
P17 & 2.661 \\
P22 & 1.242 \\
(b) Transverse direction measurement & \\
P1 & 1.262 \\
P2 & 1.004 \\
P10 & 8.502 \\
P9 & 4.757 \\
P15 & 7.133 \\
P14 & 3.324 \\
P17 & 3.120 \\
P22 & 4.085 \\
\hline
\end{tabular}

terms of surface quality. In the case of an elliptical cylinder as shown in Fig. 9, the orientation will make a difference in terms of surface quality. Before fabrication, the authors expect that part built with scanning orientation in Fig. 9a will have larger surface roughness compared to the orientation in Fig. 9b. The two fabricated elliptical parts are shown in Fig. 10a. The area marked as A and B as in Fig. 10a are aligned and a close image is taken and shown in Fig. 10b. It can be seen that the image on the right has clear staircase effect and is obviously rougher even without magnification. In general, the larger the angle between the scanning direction $r$ and the surface normal $n$ is, the rougher the surface will be. In Fig. 9, $\alpha 1$ is bigger than $\alpha 2$, so layers with scan direction in Fig. 9a will have rougher surface than that from Fig. 9b.

\subsection{Experiments with a crank and slider mechanism}

Rapid prototyping technologies have two distinct advantages: (1) build parts without geometry restriction; and (2) build subsystems (static or mobile) without the need for assembly. The first advantage has been widely discussed in previous research. The potential of the second advantage has yet to be realized even though some research has been done [16]. When fabricating a mechanism using RP technologies, the surface quality is very important in order to ensure the mechanism's mobility and accuracy. Figure 11 shows an image of a crank and slider mechanism that was fabricated in two orientations using an Objet Eden 350 machine. Based on previous studies, build orientation A should be used as cylinders will always have a better surface quality when built along its axis. But this is not true in this experiment

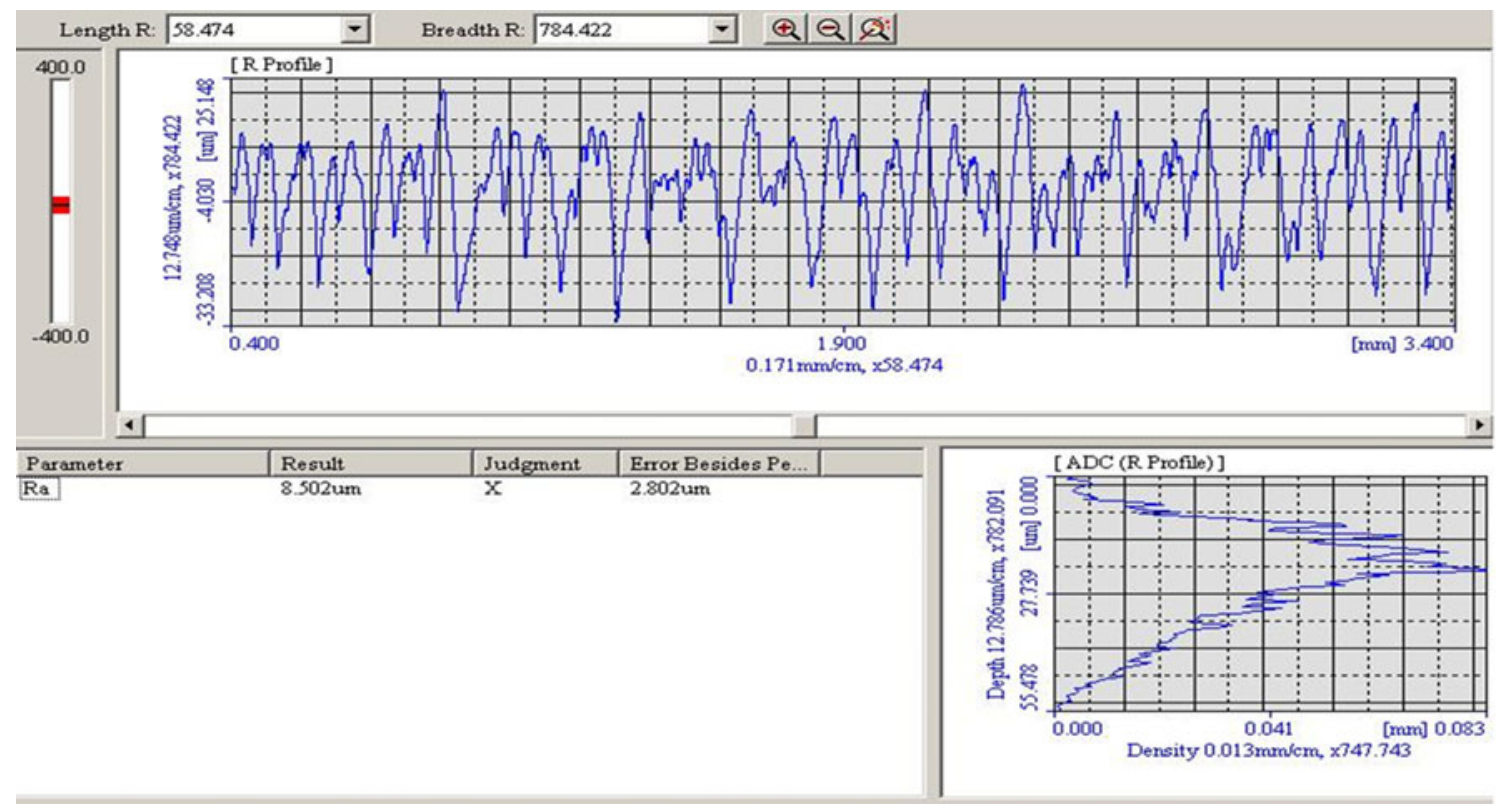

Fig. 7 Measurement Chart with the largest $R_{a}$ in transverse build orientation 
Fig. 8 Measurements for axial build orientation. a Measurement orientations b Measurement points

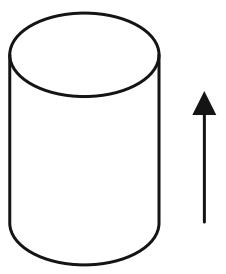

Build Orientation

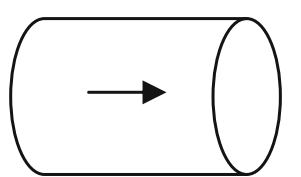

C: axial

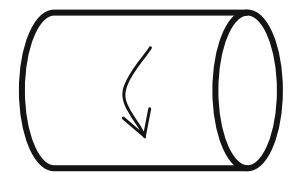

D: transverse (a) Measurement orientations

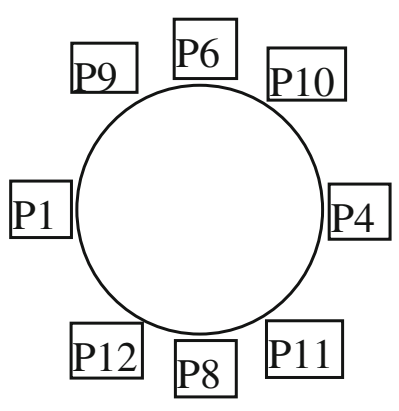

(b) Measurement points as the part built using orientation B is better. The authors have tried to build the mechanism with $0.1 \mathrm{~mm}$ joint clearance. The one built in orientation $\mathrm{A}$ cannot move while the one built in orientation B can. This experiment has again illustrated that when the layer thickness is small enough, curvature change in the build orientation has a smaller effect than the curvature change on the contours of a layer.

Table 3 Measurement results for axial build orientation

\begin{tabular}{ll}
\hline Points $\quad$ Roughness $R_{a}(\mu \mathrm{m})$ \\
\hline
\end{tabular}

$\begin{array}{lr}\text { (a) Axial direction measurement } & \\ \text { P1 } & 7.969 \\ \text { P4 } & 10.265 \\ \text { P6 } & 14.787 \\ \text { P8 } & 10.654 \\ \text { P9 } & 11.601 \\ \text { P10 } & 13.327 \\ \text { P11 } & 10.336 \\ \text { P12 } & 10.680\end{array}$

(b) Transverse direction measurements

\begin{tabular}{ll} 
P1 & 3.667 \\
P4 & 2.349 \\
P6 & 8.675 \\
P8 & 3.666 \\
P9 & 7.792 \\
P10 & 3.500 \\
P11 & 2.811 \\
P12 & 7.912 \\
\hline
\end{tabular}

\section{Analysis}

For some RP systems, the layer thickness ( $z$-axis resolution) is already very small compared to their XY positioning accuracy (resolution). For example, SLA systems from 3D systems claim to have layer thickness of $0.03 \mathrm{~mm}$ with a scanning spot size of $0.12 \mathrm{~mm}$ [13]. Objet machines have layer thickness of $0.016 \mathrm{~mm}$ and $\mathrm{XY}$ resolution of $0.042 \mathrm{~mm}$ [12]. Both the scanning spot size and XY resolution can be thought of as the road width when scanning a layer. When scanning a contour on a layer, the road width will cause staircase effect.

Figure 12 shows the staircase effect caused by a road width $h$ when scanning a layer. Suppose the contour is in circular shape and $h$ is the scanning road width. The chordal error $e$ is given by Eq. (1). It can be seen that the error will be the maximum $\mathrm{h}$ when $\alpha$ is zero. This can explain why the cylinder built in axial orientation has larger staircase error than that of along the transverse direction as shown in Fig. 3 and Table 3.

$e=h \cos \alpha$

For

Table 4 Summary of maximum $R_{a}$ in all measurements

\begin{tabular}{llr}
\hline Build orientations & $\begin{array}{l}\text { Measurement } \\
\text { direction }\end{array}$ & $\begin{array}{l}\text { Max. roughness } \\
R_{a}(\mu \mathrm{m})\end{array}$ \\
\hline Transverse orientation & Axial & 3.122 \\
& Transverse & 8.502 \\
Axial orientation & Axial & 14.787 \\
& Transverse & 8.675 \\
\hline
\end{tabular}


Fig. 9 An elliptical cylinder with two scanning orientations. a Long axis scanning. b Short axis scanning

Fig. 10 Elliptical cylinders built with different layout. a Parts built. b Close image of rough surface regions

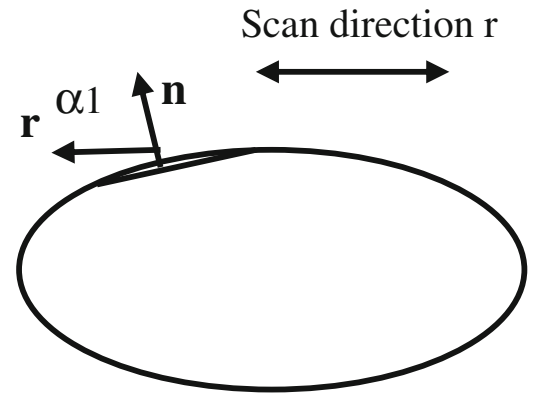

(a) Long axis scanning

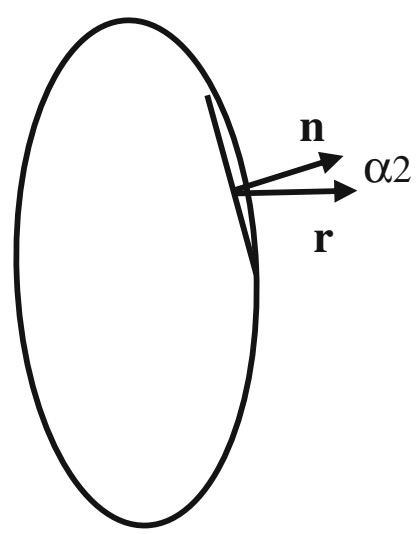

(b) Short axis scanning

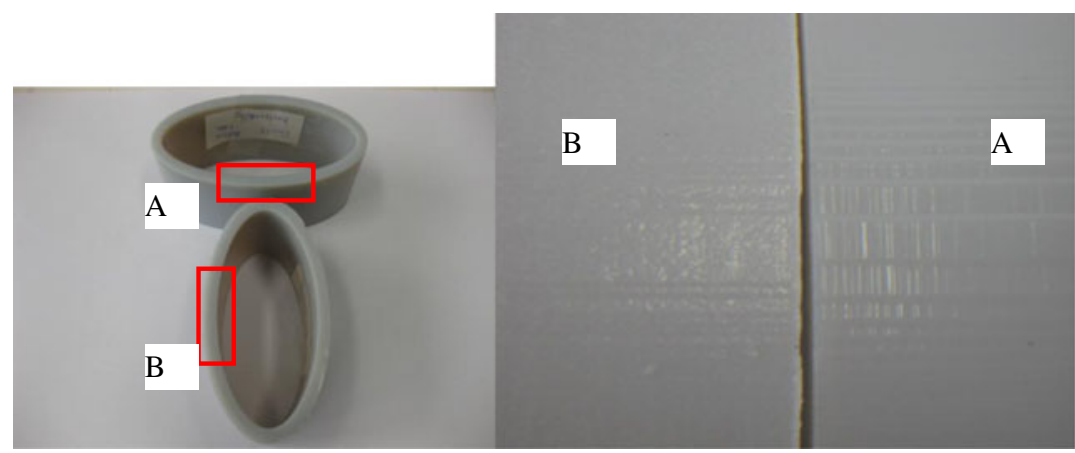

(a) Parts built

(b) close image of rough surface regions

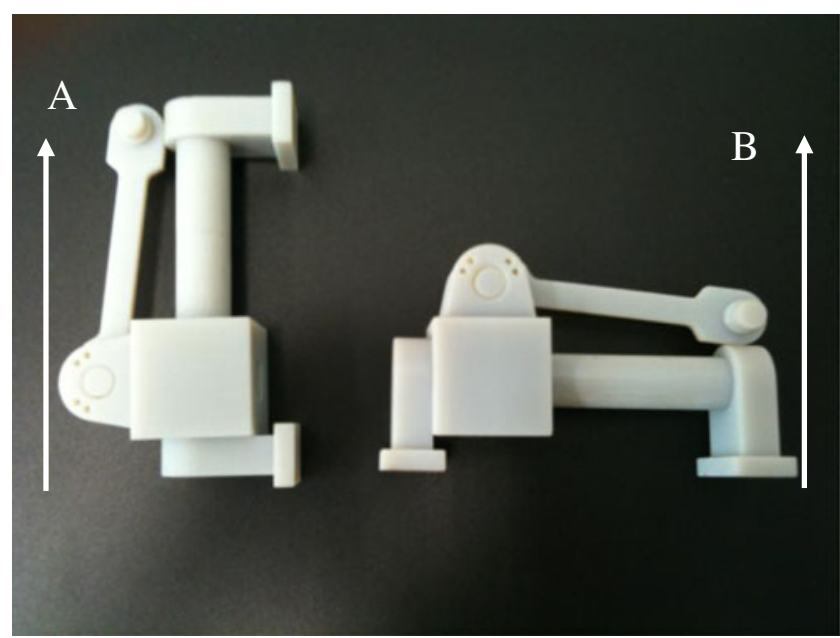

Fig. 11 Mechanism built in two orientations $\alpha \in\left[0.90^{\circ}\right]$,

In part build orientation, the expression for staircase error is the same as in Eq. (2)

$E=H \cos \beta$

For

$\beta \in\left[0.90^{\circ}\right]$

where $E$ is the staircase chordal error along the $z$ direction of an RP machine, $H$ is the layer thickness, and $\beta$ is the angle defined the same as for $\alpha$.

For the case of Objet Eden350 used in this research, $H$ is 0.016 and $h$ is 0.042 . So

$$
\begin{aligned}
& e=0.042 \cos \alpha \\
& E=0.016 \cos \beta
\end{aligned}
$$

From Eq. (3), it can be seen that the chordal error $e$ on a 

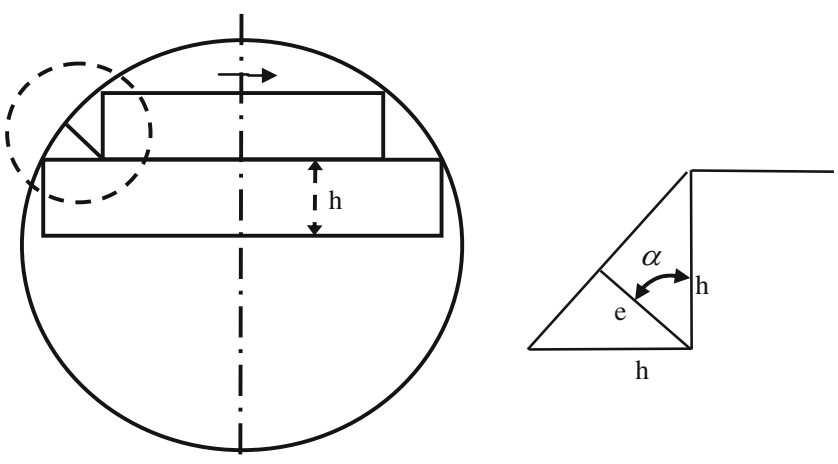

Fig. 12 Staircase effect on a layer due to road width

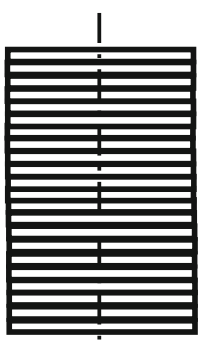

i

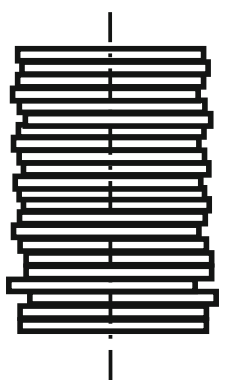

\section{(a) A theoretic (b) A real cylinder cylinder}

Fig. 13 Roughness error in axial orientation. a A theoretic cylinder. b A real cylinder layer is more sensitive to the curvature change (implied by the angle change).

Referring to Fig. 9, the dot product between the unit normal $n$ and a unit vector in the raster scanning direction $r$ can be represented as $A=n . r=|n| x|r|$ cos where is the angle between the two vectors. $A$ will be in the range $(0,|n| . \mid$ $r \mid)$. When $A$ is smaller, it is more likely to have staircase effect, thus generate a rougher surface. So in the scanning orientation, it is preferred to have less contour line segments with small $A$ values.

In the measurement summary shown in Table 4, the maximum roughness of $14.787 \mu \mathrm{m}$ is recorded when the cylinder is built in axial orientation and measured in axial orientation too. This cannot be simply explained by build orientation.

Figure 13a shows the idea case of stacking layers along the axial orientation. The actual measurements have indicated a rough surface as illustrated in Fig. 13b (exaggerated). The authors speculate that the following are the main reasons:

1. Error due to drastic scanning velocity change

This is perhaps the major contribution of the surface error. When scanning a layer, quality at the ends is extremely difficult to control. Figure 14a, shows two adjacent scanning roads. When scanning to an end-point $\mathrm{B}$, the velocity must be reduced to near zero. And at end-point $\mathrm{D}$, the velocity must be accelerated from near zero to a much larger value. The velocity change at the ends causes system oscillation, thus causes surface quality uncertainty. Figure 14b shows a possible pattern of velocity transition from point $\mathrm{B}$ to $\mathrm{D}$. It must be pointed out that the transition is normally chaotic. Error due to drastic velocity change is equally applicable to all other RP techniques such as SLA, SLS, etc.

2. Layer registration error

At the scanning ends such as A, B, C, D in Fig. 14, it is extremely difficult to control how the droplet volume
Fig. 14 Scanning on a layer. a Two adjacent scanning roads. $\mathbf{b}$ Velocity uncertainty at the ends

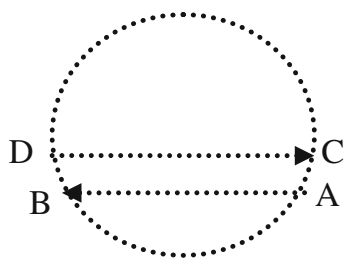

(a) Two adjacent scanning roads (b) Velocity uncertainty at the ends 
will spread. This adds to the uncertainty of surface quality at the ends.

3. XY positioning error

Compared to Z-direction accuracy, XY positioning error is normally much bigger. This causes relatively large positional errors at the ends such as A, B, C, D in Fig. 14.

From the above discussions, it is concluded that when a good surface is desired, it is recommended to avoid any scanning end points such as A, B, C, D in Fig. 14a on this surface. This can explain why the surface roughness value along the axial orientation is big for the cylinder built in the axial orientation.

\section{Conclusions}

This paper has investigated influences of part build orientation and scanning orientation to surface quality by experiments and analysis. It is found that when the layers are getting thinner, the scanning orientation on layers has a bigger impact to surface quality. Thus, it is recommended that for better surface quality, it is better to have more layer contour segments to be perpendicular or near perpendicular to the scanning orientation. It is also found that to get a better surface quality for a specific surface, it is recommended to avoid any scanning end points on this surface.

Acknowledgments The authors would like to express their gratitude to Mr Frank Tse of the Advanced Product Development Lab of HKU for help in making the prototypes and setting up the measurements. A CRCG grant from HKU is also appreciated.

Open Access This article is distributed under the terms of the Creative Commons Attribution License which permits any use, distribution, and reproduction in any medium, provided the original author(s) and the source are credited.

\section{References}

1. Gibson I, Rosen DW, Stucker B (2010) Additive manufacturing technologies: rapid prototyping to direct digital manufacturing. Springer, New York

2. Cheng W, Fuh JYH, Nee AYC, Wong YS, Loh HT, Miyazawa T (1995) Multi-objective optimization of part building orientation in stereolithography. Rapid Prototyping J 1(4):12-23

3. Fadel GM, Kirschman C (1996) Accuracy issues in CAD to RP translation. Rapid Prototyping J 2(2):4-17

4. Reeves PE, Cobb RC (1997) Reducing the surface deviation of stereolithography using in-process techniques. Rapid Prototyping J 3(1):20-31

5. Hur J, Lee K (1998) The development of a CAD environment to determine the preferred build-up direction for layered manufacturing. Int J Adv Manuf Technol 14(4):247-254

6. Pham DT, Dimov DT, Gault RS (1999) Part orientation in stereolithography. Int J Adv Manuf Technol 15(9):674-682

7. Xu F, Loh HT, Wong YS (1999) Considerations and selection of optimal orientation for different rapid prototyping systems. Rapid Prototyping J 5(2):54-60

8. Campbell RI, Artorelli M, Lee HS (2002) Surface roughness visualization for rapid prototyping models. Comput Aided Des 34:717-725

9. Kulkarni P, Marsan A, Dutta D (2000) A review of process planning techniques in layered manufacturing. Rapid Prototyping J 6(1):18-35

10. Byun HS, Lee KH (2006) Determination of the optimal build direction for different rapid prototyping processes using multi-criterion decision making. Robot Comput Integrated Manuf 22:69-80

11. Ahn DK, Kim HC, Lee SH (2007) Fabrication direction optimization to minimize post-machining in layered manufacturing. Int $\mathrm{J}$ Mach Tool Manuf 47(3-4):593-606

12. Galantucci LM, Lavecchia F, Percoco G (2009) Experimental study aiming to enhance the surface finish of fused deposition modeled parts. CIRP Ann Manuf Technol 58:189-192

13. Canellidis V, Giannatsis J, Dedoussis V (2009) Genetic-algorithmbased multi-objective optimization of the build orientation in stereolithography. Int J Adv Manuf Technol 45:714-730

14. Objet Geometries. http://www.objet.com/. Accessed in December 2011

15. 3D Systems. http://www.3dsystems.com/. Accessed in December 2011

16. Chen YH, Chen ZZ (2011) Joint analysis in rapid fabrication of non-assembly mechanisms. Rapid Prototyping J 17(6):408-417 